\title{
Hemodynamic Profiles and Their Prognostic Relevance in Cardiac Amyloidosis
}

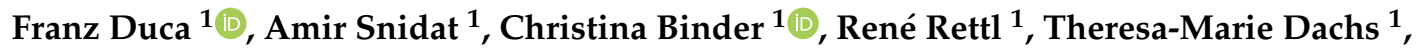 \\ Benjamin Seirer ${ }^{1}$ D, Luciana Camuz-Ligios ${ }^{1}$, Fabian Dusik ${ }^{1}$, Christophe Denis Josef Capelle ${ }^{1}$, \\ Qin Hong ${ }^{1}$, Hermine Agis ${ }^{2}$, Renate Kain ${ }^{3}$, Julia Mascherbauer ${ }^{1}$, Christian Hengstenberg ${ }^{1}$ (D), \\ Roza Badr Eslam 1,* and Diana Bonderman 1,*
}

1 Department of Internal Medicine II, Department of Cardiology, Medical University of Vienna, 1090 Vienna, Austria; franz.duca@meduniwien.ac.at (F.D.); amir.snidat@meduniwien.ac.at (A.S.); christina.binder@meduniwien.ac.at (C.B.); rene.rettl@meduniwien.ac.at (R.R.); theresa-marie.dachs@meduniwien.ac.at (T.-M.D.); benjamin.seirer@meduniwien.ac.at (B.S.); luciana.camuzligios@meduniwien.ac.at (L.C.-L.); fabian.dusik@meduniwien.ac.at (F.D.); christophe.capelle@meduniwien.ac.at (C.D.J.C.); hong.qin@akhwien.at (Q.H.); julia.mascherbauer@meduniwien.ac.at (J.M.); christian.hengstenberg@meduniwien.ac.at (C.H.)

2 Department of Internal Medicine I, Department of Oncology, Medical University of Vienna, 1090 Vienna, Austria; hermine.agis@meduniwien.ac.at

3 Clinical Institute of Pathology, Medical University of Vienna, 1090 Vienna, Austria; renate.agis@meduniwien.ac.at

* Correspondence: roza.badreslam@meduniwien.ac.at (R.B.E.); diana.bonderman@meduniwien.ac.at (D.B.); Tel.: +43-140-4004-6140 (D.B.); Fax: +43-140-4004-2160 (D.B.)

Received: 11 March 2020; Accepted: 7 April 2020; Published: 11 April 2020

\begin{abstract}
This study sought to characterize cardiac amyloidosis (CA) patients with respect to hemodynamic parameters and asses their prognostic impact in different CA cohorts. Intracardiac and pulmonary arterial pressures (PAPs) are among the strongest predictors of outcomes in patients with heart failure (HF). Despite that, the hemodynamic profiles of patients with CA and their relation to prognosis have rarely been investigated. Invasive hemodynamic, clinical, and laboratory assessment, as well as cardiac magnetic resonance imaging were performed in our CA cohort. A total of 61 patients, 35 (57.4\%) with wild-type transthyretin amyloidosis (ATTRwt) and 26 (42.6\%) with light-chain amyloidosis (AL) were enrolled. ATTRwt patients had lower N-terminal prohormone of brain natriuretic peptide values and were less frequently in New York Heart Association class $\geq$ III. Intracardiac and PAPs were elevated, but hemodynamic parameters did not differ between CA groups. Whereas in ATTRwt, the median mean PAP (hazard ratio (HR): 1.130, $p=0.040$ ) and pulmonary vascular resistance (HR: 1.010, $p=0.046$ ) were independent predictors of outcome, no hemodynamic parameter was associated with outcome in the AL group. Cardiac ATTRwt and AL patients feature elevated intracardiac and PAPs and show similar hemodynamic profiles. However, hemodynamic parameters are of greater prognostic relevance in ATTRwt, potentially providing a new therapeutic target.
\end{abstract}

Keywords: cardiac amyloidosis; pulmonary arterial pressures; invasive hemodynamics; outcomes

\section{Introduction}

Once deemed an ultra-rare disease, cardiac amyloidosis (CA) is now considered an important etiology in the diagnostic work-up of heart failure (HF) and an increasing number of cardiologists are confronted with the difficult task of treating these patients [1,2]. In HF, irrespective of left ventricular ejection fraction (LVEF), intracardiac filling pressures and pulmonary arterial pressures (PAPs) play 
a central pathophysiological role and are among the strongest predictors of adverse outcomes [3,4]. Supporting the importance of hemodynamics in HF, the recently published phase III VerICiguaT Global Study in Subjects with Heart Failure With Reduced Ejection Fraction (VICTORIA) trial, which tested vericiguat, a soluble guanylate cyclase stimulator that acts as a vasodilator in the pulmonary and systemic circulation, has met the primary endpoint i.e., the time to the first occurrence of cardiovascular death or HF hospitalization [5].

Contrary to HF, data on hemodynamics in CA are rare, stem from retrospective studies and provide conflicting results [6,7]. Therefore, further research is warranted to investigate hemodynamic profiles in different CA subgroups and to assess whether intracardiac filling pressures and PAPs are associated with patient outcomes, thus providing potential new therapeutic targets in CA.

Hence, we aimed to characterize CA patients with respect to invasively measured hemodynamic parameters and assess their prognostic relevance in a prospectively followed cohort consisting of wild-type transthyretin amyloidosis (ATTRwt) and light-chain amyloidosis (AL) patients.

\section{Materials and Methods}

\subsection{Setting and Study Design}

The present study was performed within the scope of a prospective HF registry at the Department of Internal Medicine II, Division of Cardiology at the Medical University of Vienna, which includes a dedicated amyloidosis outpatient clinic. The study was approved by the local ethics committee (EK\# 796/2010) and conducted according to good clinical practice as outlined in the declaration of Helsinki. All patients gave written informed consent.

\subsection{Clinical Definitions}

\subsubsection{Diagnosis of Cardiac Transthyretin Amyloidosis}

Until June 2016, cardiac transthyretin amyloidosis (ATTR) was diagnosed if endomyocardial biopsy (EMB) samples stained positive for Congo red, showed apple green birefringence under polarized light and reacted with anti-TTR antibodies during immunohistochemical staining. Following the publication of Gillmore and colleagues in June 2016, who provided an algorithm that allows a non-biopsy diagnosis of cardiac ATTR [8], obtaining EMBs became only necessary if non-invasive test results, including cardiac magnetic resonance imaging (CMRi), transthoracic echocardiography (TTE), bone scintigraphy, serum immunofixation, urine immunofixation, and serum free light-chain assay were ambiguous. If cardiac ATTR, irrespective of diagnostic algorithm, was diagnosed, patients were offered sequencing of the TTR gene.

\subsubsection{Diagnosis of Cardiac Light-Chain Amyloidosis}

Cardiac AL was diagnosed if EBM or extra-myocardial biopsy samples stained positive for Congo red, showed apple green birefringence under polarized light and reacted with light-chain antibodies during immunohistochemical staining. In order to confirm cardiac involvement in the case of extra-myocardial biopsy, we complied with current recommendations [9].

\subsubsection{Obtaining and Histological Analysis of Biopsy Samples}

EMB specimens were acquired from the left ventricle during left heart catheterization (Bipal ${ }^{\circledR}$ biopsy forceps, Cordis ${ }^{\circledR}$ Corporation, Bridgewater, NJ, USA). All biopsy specimens, irrespective of biopsy site, were immediately fixed in $7.5 \%$ buffered formalin for $24 \mathrm{~h}$ followed by paraffin embedding. Samples were cut in $2 \mu \mathrm{m}, 3 \mu \mathrm{m}$ and $6 \mu \mathrm{m}$ sections using a Leica RM 2255 Microtome (Charleston, SC, USA). Slices of $3 \mu \mathrm{m}$ were stained with modified trichrome [10], $6 \mu \mathrm{m}$ sections were used for Congo red staining, and visualization under polarized light. Slices of $2 \mu \mathrm{m}$ were used for immunohistochemical staining (AmY-kit amyloid antibodies, Martinsried, Germany). 


\subsection{Cardiac Magnetic Resonance Imaging}

CMRi was performed on a 1.5 Tesla scanner (MAGNETOM Avanto, Siemens Healthcare GmbH, Erlangen, Germany) according to standard protocols [11], which included late gadolinium enhancement imaging $(0.1 \mathrm{mmoL} / \mathrm{kg}$ gadobutrol, Gadovist, Bayer Vital GmbH, Leverkusen, Germany) and T1 mapping using the modified Look-Locker inversion (MOLLI) sequence [11-13].

\subsubsection{Transthorathic Echocardiography}

TTE was performed by certified and experienced operators on high-end machines (GE Vivid E95 and Vivid 7; GE Healthcare, Wauwatosa, WI, USA) according to current recommendations $[14,15]$. Left-sided heart valve stenosis or regurgitation $\geq$ moderate was deemed significant.

\subsubsection{Invasive Hemodynamic Assessment}

Hemodynamic parameters were assessed during right heart catheterization with a 7F Swan-Ganz catheter (Edwards Lifesciences, Irvine, CA, USA). Average pressures over eight heart cycles were used for analysis (CathCorLX (Siemens AG, Berlin and Munich, Germany)). Directly measured parameters were mean PAP (mPAP), pulmonary artery wedge pressure (PAWP), right atrial pressure (RAP), cardiac index (CI), and stroke volume index (SVi). Further parameters of interest, which included diastolic pressure gradient and pulmonary vascular resistance (PVR), were calculated according to standard formulae [16].

\subsection{Outcome Measures}

The primary outcome measure was a combination of cardiovascular death and hospitalization for HF.

\subsection{Statistical Analysis}

Continuous variables are expressed as the median and interquartile range (IQR). Categorical variables are presented as numbers and percentages. Continuous and categorical variables were compared using the Mann-Whitney $\mathrm{U}$ test and chi-square test as appropriate. For all tests, the significance level was set to $p<0.05$.

To assess the effect of parameters of interest on event-free survival, separate uni- and multivariable Cox regression models were calculated for clinical, invasive hemodynamic, and CMRi parameters. In order to account for the limited number of events, we did not perform stepwise Cox regression analyses, but adjusted for the N-terminal prohormone of brain natriuretic peptide (NT-proBNP) and troponin $t$, which are well-established predictors of adverse outcomes in CA [17-19].

Kaplan-Meier plots (log rank test) were used to verify the time-dependent discriminative power of parameters of interest. Spearman correlation coefficients were used for correlation analyses. International Business Machines Corporation (IBM) SPSS version 26.0 (IBM Corp. Chicago, United States of America) was used for all statistical analyses.

\section{Results}

\subsection{Baseline Characteristics and Clinical Presentation of the Overall Cohort}

Between March 2012 and January 2019, 170 patients with CA were included into our prospective registry. Of those, 109 had to be excluded from our analysis, the main reason being unwillingness to undergo invasive hemodynamic assessment. A detailed patient flowchart is provided in Figure 1. 


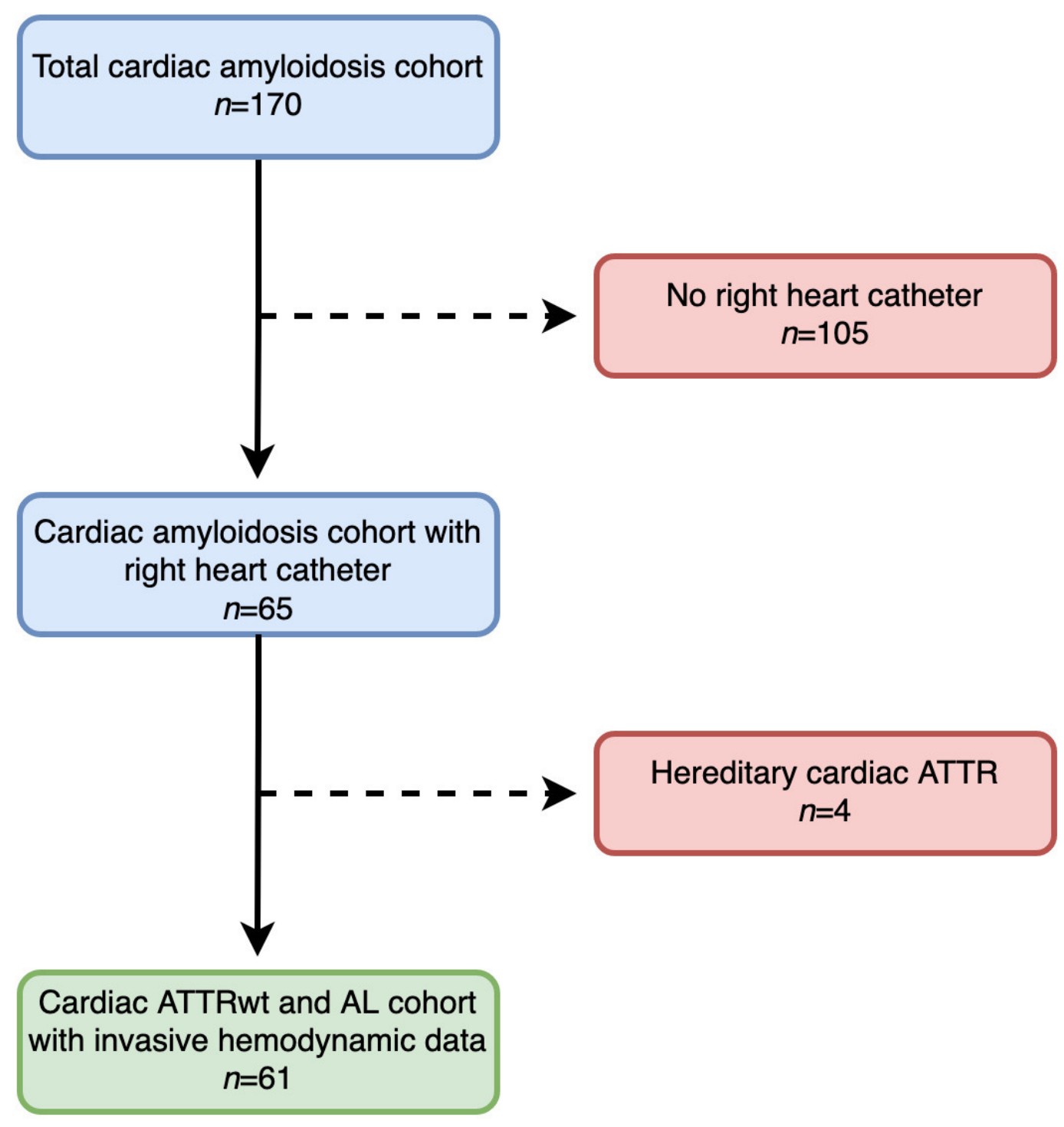

Figure 1. Patient flow-chart. A total of 170 patients were screened for the study. Reasons for exclusion are displayed. Transthyretin amyloidosis (ATTR); wild-type transthyretin amyloidosis (ATTRwt); light-chain amyloidosis (AL).

Eventually, $61 \mathrm{CA}$ patients who underwent invasive hemodynamic assessment were eligible for final data analysis, of whom $35(57.4 \%)$ patients were diagnosed with ATTRwt and $26(42.6 \%)$ with AL. Baseline characteristics are shown in Table 1. 
Table 1. Baseline characteristics.

\begin{tabular}{|c|c|c|c|c|}
\hline Variable & $\begin{array}{l}\text { All Patients } \\
\quad(n=61)\end{array}$ & $\begin{array}{l}\text { ATTRwt } \\
(n=35)\end{array}$ & $\begin{array}{c}\mathrm{AL} \\
(n=26)\end{array}$ & $\begin{array}{c}p \\
\text { Value }\end{array}$ \\
\hline \multicolumn{5}{|l|}{ Clinical parameters } \\
\hline Age, years (IQR) & $74.0(66.5-78.0)$ & $75.0(73.0-82.0)$ & $68.0(54.8-74.3)$ & $<0.001$ \\
\hline Sex, male gender, $n(\%)$ & $38.0(62.3)$ & $28.0(80.0)$ & $10.0(38.5)$ & 0.001 \\
\hline NYHA functional class $\geq$ III, $n(\%)$ & $30.0(49.2)$ & $14(40.0)$ & $16.0(61.5)$ & 0.027 \\
\hline Systolic blood pressure, $\mathrm{mmHg}$ (IQR) & $118(112-139)$ & $122(114-135)$ & $117(103-142)$ & 0.431 \\
\hline Diastolic blood pressure, $\mathrm{mmHg}$ (IQR) & $68.0(61.0-78.0)$ & $67.0(61.0-74.5)$ & $70.0(63.8-78.3)$ & 0.521 \\
\hline NT-proBNP, pg/mL (IQR) & $\begin{array}{c}3552 \\
(1501-7357)\end{array}$ & $\begin{array}{c}2368 \\
(1331-5834)\end{array}$ & $\begin{array}{c}4900 \\
(2045-10571)\end{array}$ & 0.020 \\
\hline Troponin t, ng/mL (IQR) & $0.07(0.03-0.12)$ & $0.07(0.03-0.10)$ & $0.07(0.03-0.21)$ & 0.611 \\
\hline eGFR, mL/min/1.73 m² (IQR) & $58.4(43.8-74.8)$ & $56.4(43.5-79.6)$ & $61.2(46.2-71.3)$ & 0.838 \\
\hline Combined endpoint, $n(\%)$ & $30.0(49.2)$ & $10.0(28.6)$ & $20.0(76.9)$ & $<0.001$ \\
\hline \multicolumn{5}{|l|}{ Medication } \\
\hline Beta Blocker, $n(\%)$ & $34.0(55.7)$ & $19.0(54.3)$ & $15.0(57.7)$ & 0.889 \\
\hline ACE inhibitor, $n(\%)$ & $14.0(23.0)$ & $8.0(22.9)$ & $6.0(23.1)$ & 0.967 \\
\hline Angiotensin receptor blocker, $n(\%)$ & $17.0(27.9)$ & $11.0(31.4)$ & $6.0(23.1)$ & 0.429 \\
\hline Epigallocatechin gallate, $n(\%)$ & $20.0(32.8)$ & $20.0(57.1)$ & $0.0(0.0)$ & n.a \\
\hline Tafamidis, $n(\%)$ & $2.0(3.3)$ & $2.0(5.7)$ & $0.0(0.0)$ & n.a \\
\hline Daratumumab, $n(\%)$ & $5.0(8.2)$ & $0.0(0.0)$ & $5.0(19.2)$ & n.a \\
\hline Lenalidomide, $n(\%)$ & $1.0(1.6)$ & $0.0(0.0)$ & $1.0(3.8)$ & n.a \\
\hline Thalidomide, $n(\%)$ & $2.0(3.3)$ & $0.0(0.0)$ & $2.0(7.7)$ & n.a \\
\hline Bortezomib, $n(\%)$ & $14.0(23.0)$ & $0.0(0.0)$ & $14.0(53.8)$ & n.a \\
\hline Cyclophosphamide, (\%) & $8.0(13.1)$ & $0.0(0.0)$ & $8.0(30.8)$ & n.a \\
\hline Dexamethasone, $n(\%)$ & $14.0(23.0)$ & $0.0(0.0)$ & $14.0(53.8)$ & n.a \\
\hline Rituximab, $n(\%)$ & $1.0(1.6)$ & $0.0(0.0)$ & $1.0(3.8)$ & n.a \\
\hline Bendamustine, $n(\%)$ & $1.0(1.6)$ & $0.0(0.0)$ & $1.0(3.8)$ & n.a \\
\hline No diuretic agents, $n(\%)$ & $12.0(19.7)$ & $8.0(22.9)$ & $4.0(15.4)$ & 0.434 \\
\hline One diuretic agent, $n(\%)$ & $13.0(21.3)$ & $10.0(28.6)$ & $3.0(11.5)$ & 0.096 \\
\hline Two diuretic agents, $n(\%)$ & $28.0(45.9)$ & $13.0(37.1)$ & $15.0(57.7)$ & 0.134 \\
\hline Three diuretic agents, $n(\%)$ & $7.0(11.5)$ & $3(8.6)$ & $4.0(15.4)$ & 0.433 \\
\hline \multicolumn{5}{|l|}{ Invasive hemodynamic parameters } \\
\hline Mean pulmonary arterial pressure, $\mathrm{mmHg}$ (IQR) & $30.0(25.5-36.5)$ & $30.0(26.0-34.0)$ & $32.0(25.0-43.0)$ & 0.296 \\
\hline Right atrial pressure, $\mathrm{mmHg}(\mathrm{IQR})$ & $11.0(7.3-16.8)$ & $11.0(7.8-16.0)$ & $11.5(7.0-18.0)$ & 0.654 \\
\hline Pulmonary artery wedge pressure, $\mathrm{mmHg}$ (IQR) & $20.0(16.5-24.0)$ & $19.0(16.0-22.0)$ & $20.5(16.8-29.3)$ & 0.201 \\
\hline Cardiac index, $\mathrm{L} / \mathrm{min} / \mathrm{m}^{2}(\mathrm{IQR})$ & $2.4(1.9-2.8)$ & $2.4(2.0-2.7)$ & $2.4(1.8-3.1)$ & 0.941 \\
\hline Stroke volume index, $\mathrm{mL} / \mathrm{m}^{2}$ (IQR) & $30.7(25.2-41.6)$ & $31.4(24.4-42.3)$ & $30.3(25.9-40.2)$ & 0.835 \\
\hline Pulmonary vascular resistance, dyn $\cdot \mathrm{s} \cdot \mathrm{cm}^{-5}$ (IQR) & $180(129-266)$ & $181(128-300)$ & $166(126-264)$ & 0.726 \\
\hline Diastolic pressure gradient, $\mathrm{mmHg}(\mathrm{IQR})$ & $1.0(-1.0-3.8)$ & $2.0(-1.0-4.3)$ & $0.0(-1.3-3.0)$ & 0.217 \\
\hline \multicolumn{5}{|l|}{ Cardiac magnetic resonance imaging parameters } \\
\hline MOLLI-ECV, \% (IQR) & $47.2(41.0-55.9)$ & $48.0(41.1-55.6)$ & $45.8(39.6-65.4)$ & 0.860 \\
\hline Left atrial area, $\mathrm{cm}^{2}$ (IQR) & $31.5(26.0-37.3)$ & $32.5(27.8-38.8)$ & $30.0(24.0-31.0)$ & 0.185 \\
\hline Right atrial area, $\mathrm{cm}^{2}$ (IQR) & $29.0(24.0-38.0)$ & $33.0(27.8-39.5)$ & $25.5(24.0-31.0)$ & 0.010 \\
\hline Left ventricular ejection fraction, \% (IQR) & $57.5(50.0-66.3)$ & $55.6(49.0-60.5)$ & $62.5(52.3-67.0)$ & 0.077 \\
\hline Left ventricular end-diastolic volume index, $\mathrm{ml} / \mathrm{m}^{2}$ (IQR) & $66.1(56.5-85.0)$ & $81.6(64.9-91.5)$ & $60.1(45.9-72.3)$ & 0.001 \\
\hline Interventricular septum, mm (IQR) & $19.0(15.5-22.0)$ & $20.0(16.0-23.0)$ & $17.0(13.0-20.0)$ & 0.040 \\
\hline Right ventricular ejection fraction, \% (IQR) & $48.0(41.0-61.5)$ & $48.0(38.0-60.5)$ & $52.0(42.0-62.0)$ & 0.629 \\
\hline Right ventricular end-diastolic volume index, $\mathrm{mL} / \mathrm{m}^{2}$ (IQR) & $78.5(64.0-96.7)$ & $82.8(66.7-100)$ & $72.3(62.1-94.0)$ & 0.133 \\
\hline \multicolumn{5}{|l|}{ Transthorathic echocardiography parameters } \\
\hline Significant aortic valve stenosis, $n(\%)$ & $1.0(1.6)$ & $1.0(2.9)$ & $0.0(0.0)$ & 0.378 \\
\hline Significant aortic valve regurgitation, $n(\%)$ & $1.0(1.6)$ & $1.0(2.9)$ & $0.0(0.0)$ & 0.378 \\
\hline Significant mitral valve stenosis, $n(\%)$ & $0.0(0.0)$ & $0.0(0.0)$ & $0.0(0.0)$ & n.a \\
\hline Significant mitral valve regurgitation, $n(\%)$ & $20.0(32.8)$ & $12.0(34.3)$ & $8.0(30.8)$ & 0.713 \\
\hline
\end{tabular}

Wild-type transthyretin amyloidosis (ATTRwt); light-chain amyloidosis (AL); interquartile range (IQR); New York Heart Association (NYHA); N-terminal prohormone of brain natriuretic peptide (NT-proBNP); estimated glomerular filtration rate (eGFR); angiotensin converting enzyme (ACE); modified Look-Locker inversion recovery sequence derived extracellular volume (MOLLI-ECV). Valvular stenosis or regurgitation $\geq$ moderate was considered significant; n.a indicates not applicable. Bold indicates statistical significance.

Median NT-proBNP values of $3552 \mathrm{pg} / \mathrm{mL}$ (IQR: 1501-7357) and the fact that almost half $(n=30$, 49.2\%) of all patients were in New York Heart Association (NYHA) class $\geq$ III suggest that study 
participants were in rather advanced stages of HF. No difference with respect to HF severity could be detected between patients who underwent invasive hemodynamic assessment and patients who did not (NYHA class $\geq$ III: $n=30.0$ (49.2) \% vs. $n=38.0$ (36.2\%), $p=0.106$; median NT-proBNP: 3552 pg/mL (IQR: 1501-7357) versus $2972 \mathrm{pg} / \mathrm{mL}$ (IQR: 1238-6801), $p=0.478$ ).

Furthermore, patients showed marked elevations in pulmonary arterial and intracardiac filling pressures (mPAP: $30.0 \mathrm{mmHg}$, IQR: 25.5-36.5; RAP: $11.0 \mathrm{mmHg}$, IQR: 7.3-16.8; PAWP: $20.0 \mathrm{mmHg}$, IQR: 16.5-24.0), whereas CI (2.4 L/min/ $/ \mathrm{m}^{2}$, IQR: $\left.1.9-2.8\right)$ and SVi (30.7 mL/m², IQR: 25.2-41.6) were mostly within normal ranges.

Regarding cardiac structural and functional parameters, patients had pronounced left ventricular (LV) hypertrophy (interventricular septum (IVS): $19 \mathrm{~mm}$, IQR: 15.5-22.0) and elevated MOLLI-extracellular volume (MOLLI-ECV) values (47.2\%, IQR: 41.0-55.9). LVEF, LV end-diastolic volume index (EDVi), right ventricular (RV) EF, and RVEDVi were $57.5 \%$ (IQR: 50.0-66.3), $66.1 \mathrm{~mL} / \mathrm{m}^{2}$ (IQR: 99.8-170), 48.0\% (IQR: 41.0-61.5), and $78.5 \mathrm{~mL} / \mathrm{m}^{2}$ (IQR: 64.0-96.7), respectively.

Significant combined aortic valve disease was present in one patient $(1.6 \%)$. Relevant mitral regurgitation (MR) was present in $20(32.8 \%)$ patients.

\subsection{Differences Between Cardiac Wild-Type Transthyretin and Light-Chain Amyloidosis Patients}

We detected clear clinical differences between cardiac ATTRwt and AL patients (Table 1). ATTRwt patients were older (75.0 years, IQR: 73.0-82.0 versus 68.0 years, IQR: 54.8-74.3; $p<0.001$ ), more often male ( $n=28,80 \%$ vs. $n=10,38.5 ; p=0.001$ ), less symptomatic (NYHA class $\geq$ III: $n=14,40 \%$ versus $n=16,61.5 \% ; p=0.027)$, and had lower levels of NT-proBNP (2368 pg/mL, IQR: 1331-5834 vs. 4900 pg/mL, IQR: 2045-10,571; $p=0.020$ ).

Invasive hemodynamic parameters, however, did not differ between CA subtypes. Differences with regards to imaging parameters are provided in Table 1.

\subsection{Patient Outcomes}

During a median follow-up of 19.0 months (IQR: 3.5-32.0), 30 patients $(49.2 \%)$ reached the composite endpoint of either HF hospitalization or cardiovascular death (Table 1). Median overall survival in the total CA cohort was 49.0 months.

Compared to AL patients, ATTRwt patients experienced the endpoint less often $(n=10$, $28.6 \%$ versus $n=20,76.9 \% ; p<0.001$ ). Median overall survival was 14.0 months in the AL and 53.0 months in the ATTRwt cohorts.

Uni- and multivariable Cox regression analyses and Kaplan-Meier curves for the total CA cohort are shown in Table S1 and Figure S1.

Clinical parameters that were independently associated with outcome were age (hazard ratio (HR): $0.958,95 \%$ confidence interval (CI): 0.924-0.993; $p=0.020$ ), male gender (HR: 0.277, 95\% CI: $0.122-0.628 ; p=0.002$ ), NYHA class $\geq$ III (HR: $3.214,95 \%$ CI: $1.211-8.529 ; p=0.019$ ), NT-proBNP (HR: $1.940,95 \%$ CI: 1.327-2.838; $p=0.001$ ), and troponin t (HR: 1.558, 95\% CI: $1.073-2.264 ; p=0.020$ ). Among hemodynamic parameters mPAP (HR: 1.034, 95\% CI: 1.001-1.069; $p=0.045)$ and PAWP (HR: 1.073, 95\% CI: 1.011-1.139; $p=0.021)$ remained predictors of outcome in our multivariable model. LVEDVi (HR: 0.972, 95\% CI: 0.952-0.992; $p=0.007$ ) was the only CMRi parameter predictive of adverse events. No association between patient outcomes and concomitant valvular heart disease was found [aortic stenosis (AS): HR: 1.072, 95\% CI: 0.142-8.109; $p=0.946$; aortic regurgitation (AR): HR: 1.072, 95\% CI: 0.142-8.109; $p=0.946$, MR: HR: $1.116,95 \%$ CI: 0.529-2.353; $p=0.773$ ).

\subsection{Predictors of Outcome in Cardiac Wild-Type Transthyretin Amyloidosis Patients}

Uni- and multivariable Cox regression analyses for ATTRwt patients are shown in Table 2. Among clinical parameters, age (HR: 1.255, 95\% CI: 1.055-1.493; $p=0.010$ ], diastolic blood pressure (HR: $0.897,95 \%$ CI: $0.828-0.971 ; p=0.008$ ), troponin $\mathrm{t}$ (HR: 2.466, 95\% CI: $1.187-5.124 ; p=0.016$ ), and the 
number of diuretic agents (HR: 5.932, 95\% CI: 1.217-28.90; $p=0.028$ ) were independent predictors of adverse patient outcomes.

Among invasive hemodynamic parameters, mPAP (HR: 1.130, 95\% CI: 1.006-1.269; $p=0.040$ ) and PVR (HR: 1.010, 95\% CI: 1.000-1.020; $p=0.046$ ) were associated with outcomes in the multivariable analyses.

MOLLI-ECV was predictive of outcomes in the univariable, but not in the multivariable analyses.

Kaplan-Meier curves (Figure 2A) and corresponding log rank test $(p=0.007)$ showed worse event-free survival for patients with $\mathrm{mPAP} \geq$ median $(30.0 \mathrm{mmHg})$.

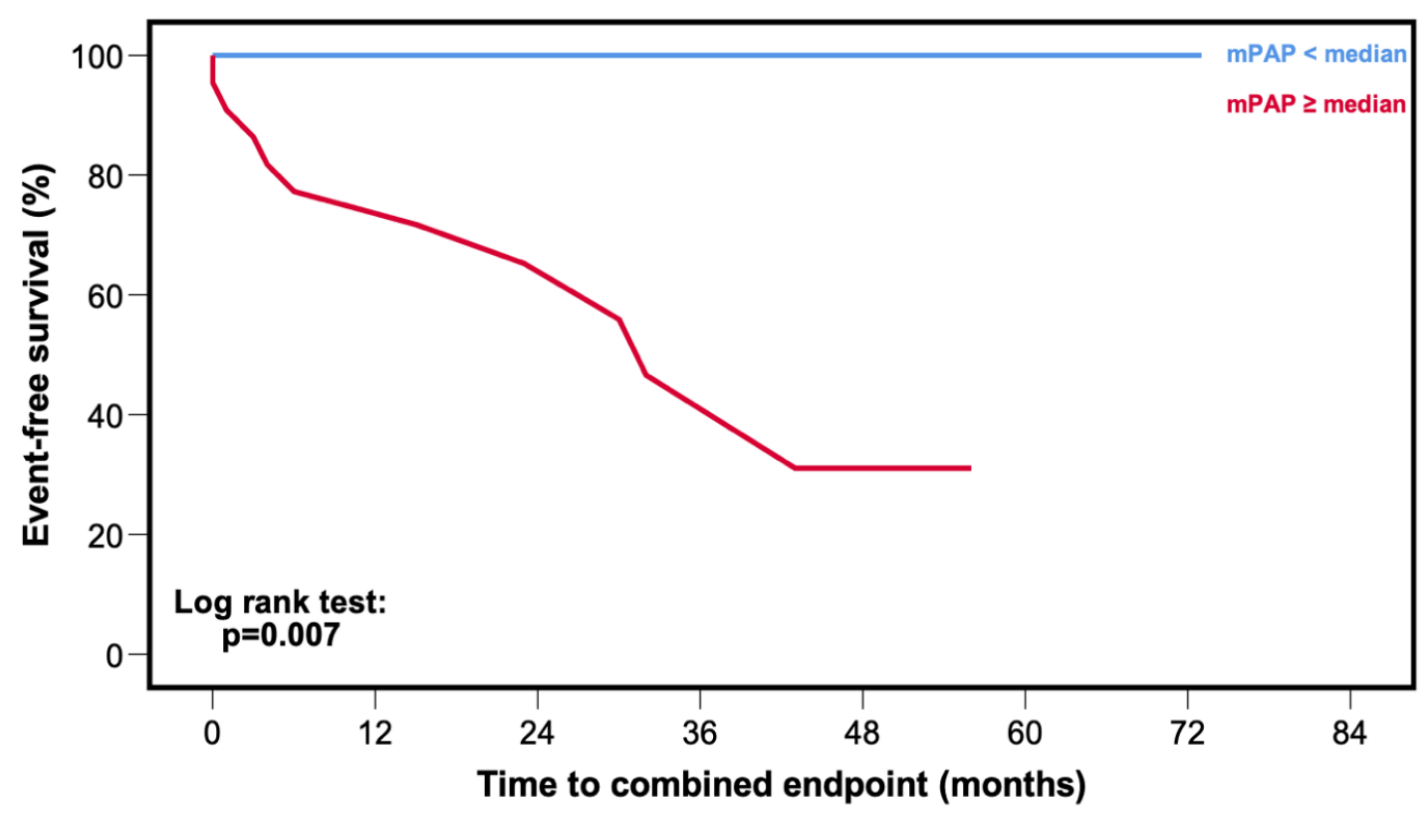

(A)

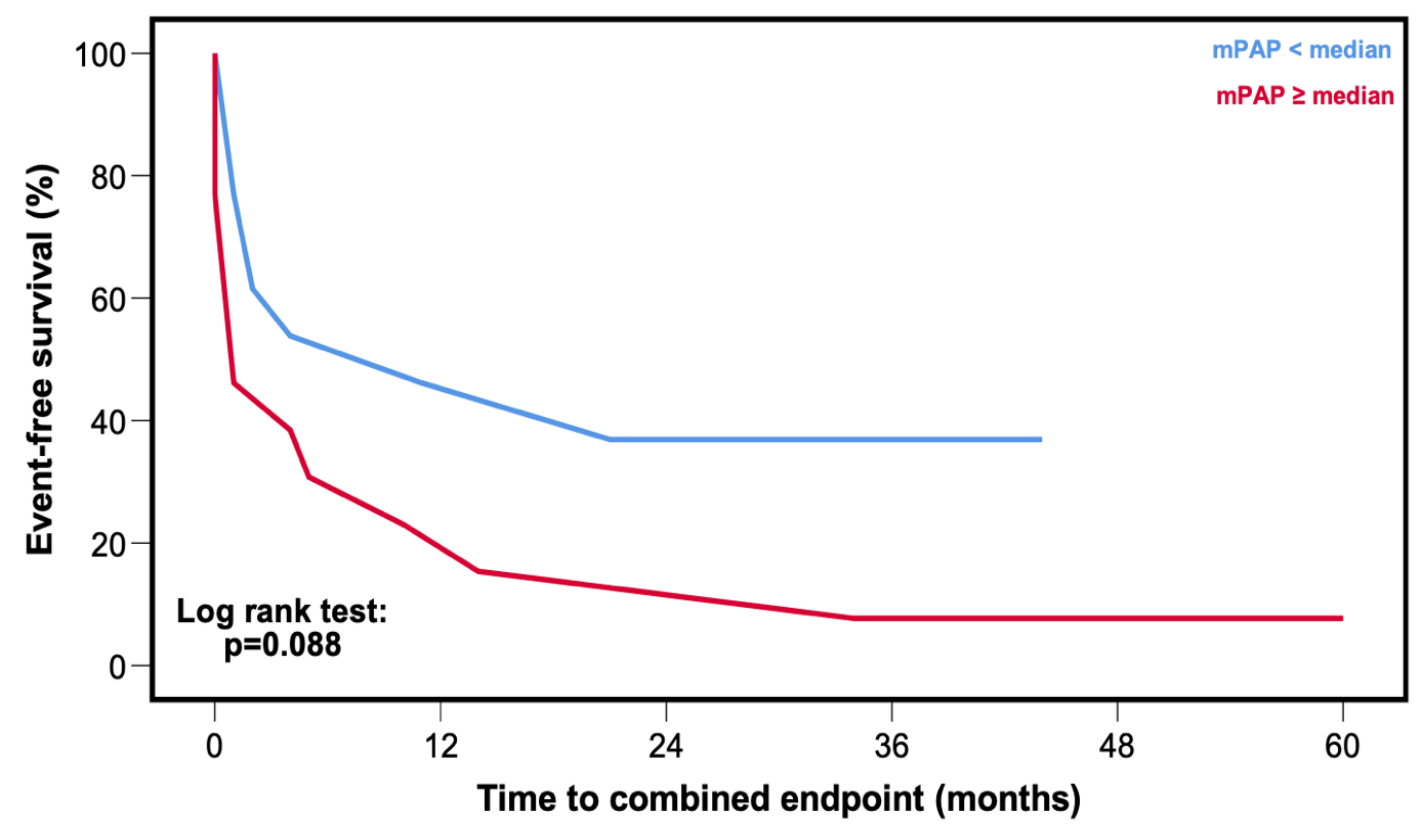

(B)

Figure 2. Kaplan-Meier curves for event-free survival stratified by median mean pulmonary arterial pressure (mPAP). (A) Patients with cardiac wild-type transthyretin amyloidosis. (B) Patients with cardiac light-chain amyloidosis. 
Table 2. Cox regression analysis for the cardiac wild-type transthyretin amyloidosis cohort.

\begin{tabular}{|c|c|c|c|c|c|c|}
\hline Variable & $\begin{array}{c}\text { Crude } \\
\text { Hazard } \\
\text { Ratio }\end{array}$ & $\begin{array}{c}95 \% \\
\text { Confidence } \\
\text { Interval }\end{array}$ & $\begin{array}{c}p \\
\text { Value }\end{array}$ & $\begin{array}{c}\text { Adjusted } \\
\text { Hazard } \\
\text { Ratio }\end{array}$ & $\begin{array}{c}95 \% \\
\text { Confidence } \\
\text { Interval }\end{array}$ & $\begin{array}{c}p \\
\text { Value }\end{array}$ \\
\hline & \multicolumn{3}{|c|}{ Univariable Regression } & \multicolumn{3}{|c|}{ Multivariable Regression } \\
\hline \multicolumn{7}{|l|}{ Clinical parameters } \\
\hline Age, years & 1.143 & $1.021-1.279$ & 0.020 & 1.255 & $1.055-1.493$ & 0.010 \\
\hline Sex, male gender & 2.252 & $0.284-17.843$ & 0.442 & 0.387 & $0.025-5.987$ & 0.497 \\
\hline NYHA functional class $\geq$ III & 1.441 & $0.379-5.485$ & 0.592 & 2.927 & $0.662-12.931$ & 0.157 \\
\hline Systolic blood pressure, $\mathrm{mmHg}$ & 0.990 & $0.955-1.027$ & 0.602 & 0.985 & $0.954-1.017$ & 0.353 \\
\hline Diastolic blood pressure, $\mathrm{mmHg}$ & 0.966 & $0.906-1.029$ & 0.283 & 0.897 & $0.828-0.971$ & 0.008 \\
\hline NT-proBNP, pg/mL* & 1.383 & $0.769-2.487$ & 0.279 & 1.181 & $0.641-2.176$ & 0.593 \\
\hline Troponin $\mathrm{t}, \mathrm{ng} / \mathrm{mL} t$ & 2.499 & $1.231-5.072$ & 0.011 & 2.466 & $1.187-5.124$ & 0.016 \\
\hline $\mathrm{eGFR}, \mathrm{mL} / \mathrm{min} / 1.73 \mathrm{~m}^{2}$ & 0.989 & $0.962-1.017$ & 0.442 & 0.991 & $0.957-1.027$ & 0.630 \\
\hline \multicolumn{7}{|l|}{ Concomitant medication } \\
\hline Beta Blocker & 0.716 & $0.187-2.733$ & 0.625 & 0.607 & $0.110-3.340$ & 0.566 \\
\hline ACE inhibitor & 0.868 & $0.173-4.347$ & 0.863 & 1.024 & $0.180-5.816$ & 0.979 \\
\hline Angiotensin receptor blocker & 1.044 & $0.260-4.184$ & 0.952 & 0.1756 & $0.391-7.893$ & 0.463 \\
\hline Number of diuretic agents & 1.276 & $0.619-2.630$ & 0.509 & 5.932 & $1.217-28.906$ & 0.028 \\
\hline \multicolumn{7}{|l|}{ Invasive hemodynamic parameters } \\
\hline Mean pulmonary arterial pressure, $\mathrm{mmHg}$ & 1.177 & $1.049-1.321$ & 0.005 & 1.130 & $1.006-1.269$ & 0.040 \\
\hline Right atrial pressure, $\mathrm{mmHg}$ & 1.124 & $0.992-1.273$ & 0.067 & 1.057 & $0.926-1.205$ & 0.411 \\
\hline Pulmonary artery wedge pressure, $\mathrm{mmHg}$ & 1.116 & $0.995-1.253$ & 0.061 & 1.045 & $0.924-1.182$ & 0.484 \\
\hline Cardiac index, $\mathrm{L} / \mathrm{min} / \mathrm{m}^{2}$ & 0.920 & $0.338-2.502$ & 0.870 & 0.656 & $0.248-1.739$ & 0.397 \\
\hline Stroke volume index, $\mathrm{mL} / \mathrm{m}^{2}$ & 0.974 & $0.920-1.031$ & 0.369 & 0.950 & $0.890-1.014$ & 0.124 \\
\hline Pulmonary vascular resistance, dyn $\cdot \mathrm{s} \cdot \mathrm{cm}^{-5}$ & 1.005 & $0.999-1.010$ & 0.086 & 1.010 & $1.000-1.020$ & 0.046 \\
\hline Diastolic pressure gradient, $\mathrm{mmHg}$ & 0.948 & $0.795-1.130$ & 0.552 & 1.036 & $0.851-1.260$ & 0.727 \\
\hline \multicolumn{7}{|l|}{$\begin{array}{c}\text { Cardiac magnetic resonance imaging } \\
\text { parameters }\end{array}$} \\
\hline MOLLI-ECV, \% & 1.064 & $1.001-1.131$ & 0.045 & 1.044 & $0.980-1.111$ & 0.181 \\
\hline Left atrial area, $\mathrm{cm}^{2}$ & 1.120 & $0.993-1.264$ & 0.065 & 1.081 & $0.940-1.241$ & 0.274 \\
\hline Right atrial area, $\mathrm{cm}^{2}$ & 1.012 & $0.927-1.104$ & 0.792 & 1.010 & $0.901-1.132$ & 0.870 \\
\hline Left ventricular ejection fraction, $\%$ & 0.981 & $0.921-1.045$ & 0.554 & 1.031 & $0.939-1.133$ & 0.524 \\
\hline $\begin{array}{l}\text { Left ventricular end-diastolic volume index, } \\
\mathrm{mL} / \mathrm{m}^{2}(\mathrm{IQR})\end{array}$ & 1.016 & $0.974-1.060$ & 0.468 & 0.984 & $0.932-1.040$ & 0.569 \\
\hline Interventricular septum, mm & 1.058 & $0.884-1.267$ & 0.536 & 1.005 & $0.851-1.188$ & 0.950 \\
\hline Right ventricular ejection fraction, $\%$ & 0.973 & $0.924-1.025$ & 0.297 & 0.990 & $0.931-1.053$ & 0.757 \\
\hline $\begin{array}{l}\text { Right ventricular end-diastolic volume index, } \\
\qquad \mathrm{mL} / \mathrm{m}^{2}(\mathrm{IQR})\end{array}$ & 1.009 & $0.993-1.026$ & 0.260 & 1.022 & $0.975-1.071$ & 0.370 \\
\hline \multicolumn{7}{|l|}{ Transthorathic echocardiography parameters } \\
\hline Significant aortic valve stenosis & 1.664 & $0.191-14.515$ & 0.645 & 5.781 & $0.417-80.052$ & 0.191 \\
\hline Significant aortic valve regurgitation & 1.664 & $0.191-14.515$ & 0.645 & 5.781 & $0.417-80.052$ & 0.191 \\
\hline Significant mitral valve regurgitation & 1.118 & $0.314-3.982$ & 0.864 & 0.230 & $0.044-1.197$ & 0.081 \\
\hline
\end{tabular}

New York Heart Association (NYHA); N-terminal prohormone of brain natriuretic peptide (NT-proBNP); estimated glomerular filtration rate (eGFR); angiotensin converting enzyme (ACE); modified Look-Locker inversion recovery sequence derived extracellular volume (MOLLI-ECV). Valvular stenosis or regurgitation $\geq$ moderate was considered significant. *NT-proBNP was graded into quartiles. †Troponin $\mathrm{T}$ was graded into quartiles. Bold indicates statistical significance.

\subsection{Predictors of Outcome in Cardiac Light-Chain Amyloidosis Patients}

Uni- and multivariable Cox regression analyses for cardiac AL patients are depicted in Table 3.

Despite the fact that a series of parameters, such as NYHA class $\geq$ III, systolic blood pressure, troponin $\mathrm{t}$, SVi, MOLLI-ECV, LVEDVi, and IVS were associated with outcomes in the univariable Cox regression model, NT-proBNP (HR: 2.856, 95\% CI: $1.277-6.388 ; p=0.011$ ) was the only parameter that remained predictive of outcomes in the multivariable Cox regression analyses.

In contrast to ATTRwt, there was no statistical difference in event-free survival among patients with $\mathrm{mPAP} \geq$ median $(32.0 \mathrm{mmHg}$ ) compared to patients with $\mathrm{mPAP}<$ median (Figure $2 \mathrm{~B}, p=0.088$ ). 
Table 3. Cox regression analysis for the cardiac light-chain amyloidosis cohort.

\begin{tabular}{|c|c|c|c|c|c|c|}
\hline Variable & $\begin{array}{c}\text { Crude } \\
\text { Hazard } \\
\text { Ratio }\end{array}$ & $\begin{array}{c}95 \% \\
\text { Confidence } \\
\text { Interval } \\
\end{array}$ & $\begin{array}{c}p \\
\text { Value }\end{array}$ & $\begin{array}{c}\text { Adjusted } \\
\text { Hazard } \\
\text { Ratio }\end{array}$ & $\begin{array}{c}95 \% \\
\text { Confidence } \\
\text { Interval }\end{array}$ & $\begin{array}{c}p \\
\text { Value }\end{array}$ \\
\hline & \multicolumn{3}{|c|}{ Univariable Regression } & \multicolumn{3}{|c|}{ Multivariable Regression } \\
\hline \multicolumn{7}{|l|}{ Clinical parameters } \\
\hline Age, years & 0.983 & $0.942-1.026$ & 0.432 & 0.972 & $0.929-1.018$ & 0.233 \\
\hline Sex, male gender & 0.632 & $0.249-1.603$ & 0.334 & 0.491 & $0.173-1.398$ & 0.183 \\
\hline NYHA functional class $\geq$ III & 14.201 & $1.833-110.010$ & 0.011 & 7.763 & $0.729-82.669$ & 0.090 \\
\hline Systolic blood pressure, $\mathrm{mmHg}$ & 0.981 & $0.962-0.999$ & 0.042 & 0.992 & $0.971-1.013$ & 0.441 \\
\hline Diastolic blood pressure, $\mathrm{mmHg}$ & 0.988 & $0.949-1.029$ & 0.568 & 0.983 & $0.949-1.018$ & 0.328 \\
\hline NT-proBNP, pg/mL * & 3.131 & $1.619-6.778$ & 0.001 & 2.856 & $1.277-6.388$ & 0.011 \\
\hline Troponin $\mathrm{t}, \mathrm{ng} / \mathrm{mL}+$ & 1.894 & $1.171-2.778$ & 0.007 & 1.180 & $0.740-1.880$ & 0.487 \\
\hline $\mathrm{eGFR}, \mathrm{mL} / \mathrm{min} / 1.73 \mathrm{~m}^{2}$ & 0.994 & $0.974-1.015$ & 0.600 & 0.978 & $0.941-1.016$ & 0.258 \\
\hline \multicolumn{7}{|l|}{ Concomitant medication } \\
\hline Beta Blocker & 1.106 & $0.444-2.756$ & 0.828 & 1.132 & $0.408-3.139$ & 0.811 \\
\hline ACE inhibitor & 1.540 & $0.554-4.279$ & 0.408 & 3.039 & $0.826-11.178$ & 0.094 \\
\hline Angiotensin receptor blocker & 0.660 & $0.220-1.977$ & 0.457 & 1.125 & $0.351-3.608$ & 0.843 \\
\hline Number of diuretic agents & 1.185 & $0.759-1.850$ & 0.455 & 1.456 & $0.777-2.728$ & 0.241 \\
\hline \multicolumn{7}{|l|}{ Invasive hemodynamic parameters } \\
\hline Mean pulmonary arterial pressure, $\mathrm{mmHg}$ & 1.027 & $0.991-1.063$ & 0.146 & 0.999 & $0.962-1.038$ & 0.970 \\
\hline Right atrial pressure, $\mathrm{mmHg}$ & 1.027 & $0.971-1.087$ & 0.348 & 1.020 & $0.956-1.089$ & 0.553 \\
\hline Pulmonary artery wedge pressure, $\mathrm{mmHg}$ & 1.060 & $0.993-1.131$ & 0.081 & 1.039 & $0.966-1.118$ & 0.302 \\
\hline Cardiac index, $\mathrm{L} / \mathrm{min} / \mathrm{m}^{2}$ & 0.608 & $0.329-1.125$ & 0.113 & 0.859 & $0.408-1.809$ & 0.690 \\
\hline Stroke volume index, $\mathrm{mL} / \mathrm{m}^{2}$ & 0.958 & $0.920-0.997$ & 0.034 & 0.987 & $0.944-1.032$ & 0.579 \\
\hline Pulmonary vascular resistance, dyn $\cdot \mathrm{s} \cdot \mathrm{cm}^{-5}$ & 1.001 & $0.999-1.003$ & 0.263 & 1.000 & $0.997-1.002$ & 0.645 \\
\hline Diastolic pressure gradient, $\mathrm{mmHg}$ & 1.042 & $0.965-1.126$ & 0.292 & 0.978 & $0.891-1.073$ & 0.635 \\
\hline \multicolumn{7}{|l|}{$\begin{array}{c}\text { Cardiac magnetic resonance imaging } \\
\text { parameters }\end{array}$} \\
\hline MOLLI-ECV, \% & 1.042 & $1.011-1.073$ & 0.007 & 1.012 & $0.970-1.055$ & 0.590 \\
\hline Left atrial area, $\mathrm{cm}^{2}$ & 0.956 & $0.902-1.012$ & 0.123 & 1.000 & $0.894-1.118$ & 0.993 \\
\hline Right atrial area, $\mathrm{cm}^{2}$ & 0.943 & $0.876-1.016$ & 0.122 & 1.004 & $0.919-1.097$ & 0.922 \\
\hline Left ventricular ejection fraction, $\%$ & 1.013 & $0.975-1.054$ & 0.504 & 1.018 & $0.974-1.065$ & 0.425 \\
\hline $\begin{array}{l}\text { Left ventricular end-diastolic volume index, } \\
\qquad \mathrm{mL} / \mathrm{m}^{2}(\mathrm{IQR})\end{array}$ & 0.977 & $0.955-0.999$ & 0.041 & 0.975 & $0.946-1.004$ & 0.090 \\
\hline Interventricular septum, mm & 1.209 & $1.057-1.384$ & 0.006 & 1.099 & $0.948-1.273$ & 0.211 \\
\hline Right ventricular ejection fraction, $\%$ & 1.003 & $0.964-1.044$ & 0.879 & 1.027 & $0.978-1.078$ & 0.287 \\
\hline $\begin{array}{l}\text { Right ventricular end-diastolic volume index, } \\
\qquad \mathrm{mL} / \mathrm{m}^{2}(\mathrm{IQR})\end{array}$ & 0.980 & $0.955-1.006$ & 0.133 & 0.989 & $0.962-1.016$ & 0.428 \\
\hline \multicolumn{7}{|l|}{ Transthorathic echocardiography parameters } \\
\hline Significant mitral valve regurgitation & 1.477 & $0.572-3.812$ & 0.420 & 1.117 & $0.374-3.332$ & 0.843 \\
\hline
\end{tabular}

New York Heart Association (NYHA); N-terminal prohormone of brain natriuretic peptide (NT-proBNP); estimated glomerular filtration rate (eGFR); angiotensin converting enzyme (ACE); modified Look-Locker inversion recovery sequence derived extracellular volume (MOLLI-ECV). Valvular stenosis or regurgitation $\geq$ moderate was considered significant. * NT-proBNP was graded into quartiles. + Troponin T was graded into quartiles. Bold indicates statistical significance.

\subsection{Comparison of Outcomes in Cardiac Wild-Type Transthyretin and Light-Chain Amyloidosis}

In a further analysis, we compared ATTRwt patients with $\mathrm{mPAP} \geq$ and $<$ median against AL patients with $\mathrm{mPAP} \geq$ and $<$ the median and could detect significant differences in event-free survival (graphical abstract, $p<0.001$ ). AL patients with mPAP $\geq$ median had the worst event-free survival, whereas ATTRwt patients with $\mathrm{mPAP}<$ median had the best event-free survival. Interestingly, there was no difference in event-free survival between patients with ATTRwt and median $\geq \mathrm{mPAP}$ and AL patients $<$ median mPAP. 


\subsection{Association of Pulmonary Arterial Pressures with Valvular Heart Disease and Diuretics}

In the total CA cohort, there was no association between the presence of valvular heart disease and mPAP or PAWP $(\mathrm{r}=0.008, p=0.954$ for AS and mPAP; $\mathrm{r}=0.064, p=0.627$ for AS and PAWP; $\mathrm{r}=0.008, p=0.954$ for AR and mPAP; $\mathrm{r}=0.064, p=0.627$ for AR and PAWP; $\mathrm{r}=0.110, p=0.401$ for MR and $\mathrm{mPAP} ; \mathrm{r}=0.216, p=0.098$ for MR and PAWP). However, a clear association between the number of diuretic agents $(r=0.342, p=0.007)$ and mPAP levels could be detected.

\section{Discussion}

The present study assessed the prognostic relevance of intracardiac and PAPs in a well-characterized, prospectively followed cohort of CA patients. The major findings of our study were that (1) ATTRwt and AL patients do not differ with respect to hemodynamics, but (2) hemodynamic parameters are of greater prognostic importance for ATTRwt patients compared to AL.

\subsection{Hemodynamic Profiles in Cardiac Amyloidosis}

Despite their prognostic significance in other types of HF, such as HF with preserved and reduced LVEF, data on invasively measured hemodynamics in CA patients are scarce $[6,20,21]$.

In line with our results, a retrospective study by Russo et al. could detect similar hemodynamics between cardiac ATTR and AL patients [6]. Furthermore, mean intracardiac filling pressures were comparable in their and our CA patient cohorts. In contrast to our study, they found that RAP was the strongest predictor of adverse patient outcomes. One possible explanation for this difference might simply be the fact that mPAP, the strongest outcome predictor among hemodynamic parameters in our series, was not measured in the aforementioned study [6]. Also, they did not perform separate survival analyses for CA subgroups.

In a further study, hemodynamic profiles differed between AL and ATTR patients, with lower RAP and PAWP values in the ATTR group [7]. However, compared with our cohort, ATTR patients were in less advanced stages of the disease, as suggested by less pronounced LV hypertrophy.

\subsection{Pulmonary Arterial Pressures as a Potential Therapeutic Target in Cardiac Amyloidosis}

One of the major findings in the present study was the difference in the prognostic relevance of mPAP between ATTRwt and AL. In our ATTRwt patients, mPAP was the strongest predictor of outcomes among invasively obtained hemodynamic parameters (Table 2, HR: 1.130, 95\% CI: 1.006-1.269; $p=0.040)$. Interestingly, when patients were stratified according to median mPAP $(30.0 \mathrm{mmHg})$, Kaplan-Meier curves showed that not a single patient with mPAP $<$ median experienced an outcome event (Figure 2A, log rank: $p=0.007$ ). In contrast, among AL patients mPAP was neither associated with adverse outcome in the Cox regression (Table 3, HR: 1.027, 95\% CI: 0.991-1.063; $p=0.146$ ) nor in the Kaplan-Meier analysis (Figure 2B, log rank: $p=0.088$ ). Furthermore, ATTRwt patients with mPAP $\geq$ median had comparable outcomes to patients with AL and mPAP $<$ median (Graphical abstract).

Given its prognostic significance, PAPs could provide an intriguing therapeutic target in this difficult-to-treat patient population. The pathophysiological mechanism behind CA is the deposition of amyloid in the cardiac extracellular matrix. This leads to the thickening, stiffening, and subsequent diastolic dysfunction of the LV. The deterioration of LV diastolic function then causes a rise in left-sided intracardiac filling pressures and PAPs, which increases the afterload posed on the RV. A reduction of PAPs decreases the load on the RV, thus improving RV function, which in turn is strongly associated with prognosis in cardiac ATTR, but not AL patients [22]. However, this hypothesis needs to be tested in interventional trials. A potential class of drugs that might be used in this setting could be soluble guanylate cyclase stimulators, which are successfully used in the treatment of precapillary forms of pulmonary hypertension, but can be administered safely in HF, and as suggested by a small case series from our study group, also in cardiac ATTR patients [23-25]. However, one major limitation is that this 
class of drugs does not stop amyloid from being produced or expedite its removal, thus not targeting the underlying pathophysiological mechanism in CA.

In a recently published phase III trial, administration of Tafamidis, a stabilizer of the TTR tetramer and one of the most promising drugs in cardiac ATTR, significantly reduced all-cause mortality as well as cardiovascular-related hospitalizations [26]. Of note was the fact that survival curves only started to diverge at 18 months of treatment. Therefore, optimizing PAPs could have an additional effect as more patients reach the timepoint where the survival benefits of amyloid-specific therapies become evident.

\subsection{Association of Diuretics with Pulmonary Arterial Pressures and Outcome}

We observed that the number of diuretics was correlated with mPAP $(\mathrm{r}=0.342, p=0.007)$ and was predictive of outcome in ATTRwt (HR: 5.932, 95\% CI: 1.217-28.906; $p=0.028$ ), but not in the AL cohort (HR: $1.456,95 \%$ CI: $0.777-2.720, p=0.241$ ). As the use of diuretics represents a surrogate for overhydration and more advanced HF stages, our findings are in line with earlier studies showing that, in contrast to AL, the most common mode of death in ATTRwt patients is cardiovascular [27].

\subsection{Limitations}

Several limitations are inherent to the present study, such as the single-center design. However, this study design allows for constant diagnostic work-up and clinical follow-up of patients. Moreover, the invasive design of our study might have led to the selection of patients in more advanced stages of HF. Nevertheless, there was no difference in NYHA class or NT-proBNP values between patients who underwent invasive hemodynamic assessment to those who have declined. Further limitations are the sample size of our cohort and the limited number of outcome events, both of which do not allow extensive Cox regression analyses. Thus, our results must be interpreted with caution, as low event per variable ratios may cause type I errors and bias away from the null hypothesis. Nevertheless, the validity of our Cox regression model is supported by numerically stable hazard ratio estimates and $95 \%$ CIs. In addition, our cohort was in a rather advanced stage of HF, potentially further limiting our outcome analyses. However, the outcome of our cardiac ATTRwt and AL cohorts resemble contemporary CA cohorts from other centers [28,29]. Due to the fact that our study cohort consists only of cardiac ATTRwt and AL patients, the generalizability of our data to other forms of CA, especially hereditary ATTR, is limited. However, we decided to exclude patients with mutations in the TTR gene from our analysis ( $n=4$, Figure 1$)$ because this patient population is highly heterogenous and has a vastly different course of disease compared to ATTRwt.

\section{Conclusions}

In this prospective registry study, we investigated the hemodynamic profiles of patients with cardiac ATTRwt and AL. Intracardiac and PAPs were elevated to a similar extent in both forms of CA.

The main finding of our study, however, was that cardiac hemodynamics were strong predictors of outcomes in ATTRwt, but not in AL, thus potentially providing a new therapeutic target in these difficult-to-treat patients with whose treatment more and more cardiologists are confronted on a regular basis. Nonetheless, given the limitations, our results must be interpreted very cautiously and studies of stronger design are warranted to further evaluate the clinical importance of our findings.

Supplementary Materials: The following are available online at http://www.mdpi.com/2077-0383/9/4/1093/s1, Figure S1: Kaplan-Meier curves for event-free survival stratified by median mean pulmonary arterial pressure (mPAP) for the total cardiac amyloidosis cohort, Table S1: Cox regression analysis for the total study cohort.

Author Contributions: Conceptualization: F.D. (Franz Duca), D.B.; methodology: F.D. (Franz Duca), D.B.; software: F.D. (Franz Duca); validation: F.D. (Franz Duca); formal analysis: F.D. (Franz Duca); investigation: F.D. (Franz Duca), A.S., C.B., R.R., T.-M.D., R.B.E., B.S., L.C.-L., F.D. (Fabian Dusik), C.D.J.C., H.A., R.K., J.M., D.B.; resources: D.B., C.H.; writing—original draft preparation: F.D. (Franz Duca), D.B.; writing—review \& editing: F.D. (Franz Duca), A.S., C.B., R.R., T.D., R.B.E., B.S,. L.C.-L., F.D. (Fabian Dusik), C.D.J.C., Q.H., H.A., R.K., J.M., C.H., D.B.; visualization: F.D. (Franz Duca); supervision: D.B.; project administration: D.B.; funding acquisition: F.D. (Franz Duca), D.B. All authors have read and agreed to the published version of the manuscript. 
Funding: Medical Scientific Fund of the Mayor of the City of Vienna: 19011 Austrian Society of Cardiology: AP00778OFF.

Acknowledgments: This study received support from the Medical Scientific Fund of the Mayor of the City of Vienna (project number: 19011, to D.B.), the Austrian Society of Cardiology (project number: AP00778OFF to F.D.), a national research grant from Pfizer Corp. (to F.D.).

Conflicts of Interest: F.D. has received speaker's fees and congress support from Bayer, Novartis, and Pfizer. D.B. has received speaker's fees and honoraria for consulting from Actelion, Bayer, Boehringer Ingelheim, GSK, AOP Orphan, United Therapeutics, and Pfizer as well as research grants from Bayer. The other authors declare that there is no conflict of interest.

\section{References}

1. Pieske, B.; Tschöpe, C.; De Boer, R.A.; Fraser, A.G.; Anker, S.D.; Donal, E.; Edelmann, F.; Fu, M.; Guazzi, M.; Lam, C.S.P.; et al. How to diagnose heart failure with preserved ejection fraction: The HFA-PEFF diagnostic algorithm: A consensus recommendation from the Heart Failure Association (HFA) of the European Society of Cardiology (ESC). Eur. Heart J. 2019, 40, 3297-3317. [CrossRef] [PubMed]

2. Gilstrap, L.G.; Dominici, F.; Wang, Y.; El-Sady, M.S.; Singh, A.; Carli, M.F.D.; Falk, R.H.; Dorbala, S. Epidemiology of Cardiac Amyloidosis-Associated Heart Failure Hospitalizations Among Fee-for-Service Medicare Beneficiaries in the United States. Circ. Heart Failure 2019, 12, e005407. [CrossRef] [PubMed]

3. Zile, M.R.; Bennett, T.D.; Hajj, S.E.; Kueffer, F.J.; Baicu, C.F.; Abraham, W.T.; Bourge, R.C.; Stevenson, L.W. Intracardiac Pressures Measured Using an Implantable Hemodynamic Monitor. Circ. Heart Fail. 2017, 10, e003594. [CrossRef] [PubMed]

4. Guazzi, M.; Naeije, R. Pulmonary Hypertension in Heart Failure: Pathophysiology, Pathobiology, and Emerging Clinical Perspectives. J. Am. Coll. Cardiol. 2017, 69, 1718-1734. [CrossRef]

5. Armstrong, P.W.; Pieske, B.; Anstrom, K.J.; Ezekowitz, J.; Hernandez, A.F.; Butler, J.; Lam, C.S.P.; Ponikowski, P.; Voors, A.A.; Jia, G.; et al. Vericiguat in Patients with Heart Failure and Reduced Ejection Fraction. N. Engl. J. Med. 2020. [CrossRef]

6. Russo, C.; Green, P.; Maurer, M. The prognostic significance of central hemodynamics in patients with cardiac amyloidosis. Amyloid 2013, 20, 199-203. [CrossRef]

7. Rapezzi, C.; Merlini, G.; Quarta, C.C.; Riva, L.; Longhi, S.; Leone, O.; Salvi, F.; Ciliberti, P.; Pastorelli, F.; Biagini, E.; et al. Systemic Cardiac Amyloidoses. Circulation 2009, 120, 1203-1212. [CrossRef]

8. Gillmore, J.D.; Maurer, M.S.; Falk, R.H.; Merlini, G.; Damy, T.; Dispenzieri, A.; Wechalekar, A.D.; Berk, J.L.; Quarta, C.C.; Grogan, M.; et al. Nonbiopsy Diagnosis of Cardiac Transthyretin Amyloidosis. Circulation 2016, 133, 2404-2412. [CrossRef]

9. Gertz, M.A.; Comenzo, R.; Falk, R.H.; Fermand, J.P.; Hazenberg, B.P.; Hawkins, P.N.; Merlini, G.; Moreau, P.; Ronco, P.; Sanchorawala, V.; et al. Definition of organ involvement and treatment response in immunoglobulin light chain amyloidosis (AL): A consensus opinion from the 10th International Symposium on Amyloid and Amyloidosis, Tours, France, 18-22 April 2004. Am. J. Hematol. 2005, 79, 319-328. [CrossRef]

10. Garvey, W.; Fathi, A.; Bigelow, F.; Carpenter, B.; Jimenez, C. A combined elastic, fibrin and collagen stain. Stain Technol. 1987, 62, 365-368. [CrossRef]

11. Kammerlander, A.A.; Marzluf, B.A.; Zotter-Tufaro, C.; Aschauer, S.; Duca, F.; Bachmann, A.; Knechtelsdorfer, K.; Wiesinger, M.; Pfaffenberger, S.; Greiser, A.; et al. T1 Mapping by CMR Imaging: From Histological Validation to Clinical Implication. JACC: Cardiovasc. Imaging 2016, 9, 14-23. [CrossRef]

12. Duca, F.; Kammerlander, A.A.; Zotter-Tufaro, C.; Aschauer, S.; Schwaiger, M.L.; Marzluf, B.A.; Bonderman, D.; Mascherbauer, J. Interstitial Fibrosis, Functional Status, and Outcomes in Heart Failure With Preserved Ejection Fraction: Insights From a Prospective Cardiac Magnetic Resonance Imaging Study. Circ. Cardiovasc. Imaging 2016, 9. [CrossRef] [PubMed]

13. Kellman, P.; Wilson, J.R.; Xue, H.; Ugander, M.; Arai, A.E. Extracellular volume fraction mapping in the myocardium, part 1: Evaluation of an automated method. J. Cardiovasc. Magn. Reson. 2012, 14, 63. [CrossRef] [PubMed]

14. Lang, R.M.; Badano, L.P.; Mor-Avi, V.; Afilalo, J.; Armstrong, A.; Ernande, L.; Flachskampf, F.A.; Foster, E.; Goldstein, S.A.; Kuznetsova, T.; et al. Recommendations for cardiac chamber quantification by echocardiography in adults: An update from the American Society of Echocardiography and the European Association of Cardiovascular Imaging. J. Am. Soc. Echocardiogr. 2015, 28, 1-39.e14. [CrossRef] 
15. Picard, M.H.; Adams, D.; Bierig, S.M.; Dent, J.M.; Douglas, P.S.; Gillam, L.D.; Keller, A.M.; Malenka, D.J.; Masoudi, F.A.; McCulloch, M.; et al. American Society of Echocardiography recommendations for quality echocardiography laboratory operations. J. Am. Soc. Echocardiogr. 2011, 24, 1-10. [CrossRef]

16. Aschauer, S.; Kammerlander, A.A.; Zotter-Tufaro, C.; Ristl, R.; Pfaffenberger, S.; Bachmann, A.; Duca, F.; Marzluf, B.A.; Bonderman, D.; Mascherbauer, J. The right heart in heart failure with preserved ejection fraction: Insights from cardiac magnetic resonance imaging and invasive haemodynamics. Eur. J. Heart Fail. 2016, 18, 71-80. [CrossRef]

17. Vittinghoff, E.; McCulloch, C.E. Relaxing the rule of ten events per variable in logistic and Cox regression. Am. J. Epidemiol. 2007, 165, 710-718. [CrossRef]

18. Kumar, S.; Dispenzieri, A.; Lacy, M.Q.; Hayman, S.R.; Buadi, F.K.; Colby, C.; Laumann, K.; Zeldenrust, S.R.; Leung, N.; Dingli, D.; et al. Revised prognostic staging system for light chain amyloidosis incorporating cardiac biomarkers and serum free light chain measurements. J. Clin. Oncol. 2012, 30, 989-995. [CrossRef]

19. Kristen, A.V.; Scherer, K.; Buss, S.; Aus dem Siepen, F.; Haufe, S.; Bauer, R.; Hinderhofer, K.; Giannitsis, E.; Hardt, S.; Haberkorn, U.; et al. Noninvasive risk stratification of patients with transthyretin amyloidosis. JACC. Cardiovasc. Imaging 2014, 7, 502-510. [CrossRef]

20. Goliasch, G.; Zotter-Tufaro, C.; Aschauer, S.; Duca, F.; Koell, B.; Kammerlander, A.A.; Ristl, R.; Lang, I.M.; Maurer, G.; Mascherbauer, J.; et al. Outcome in Heart Failure with Preserved Ejection Fraction: The Role of Myocardial Structure and Right Ventricular Performance. PLoS ONE 2015, 10, e0134479. [CrossRef]

21. Miller, W.L.; Grill, D.E.; Borlaug, B.A. Clinical features, hemodynamics, and outcomes of pulmonary hypertension due to chronic heart failure with reduced ejection fraction: Pulmonary hypertension and heart failure. JACC Heart Fail 2013, 1, 290-299. [CrossRef] [PubMed]

22. Binder, C.; Duca, F.; Stelzer, P.D.; Nitsche, C.; Rettl, R.; Aschauer, S.; Kammerlander, A.A.; Binder, T.; Agis, H.; Kain, R.; et al. Mechanisms of heart failure in transthyretin vs. light chain amyloidosis. Eur. Heart J. Cardiovasc. Imaging 2019, 20, 512-524. [CrossRef] [PubMed]

23. Ghofrani, H.-A.; Galiè, N.; Grimminger, F.; Grünig, E.; Humbert, M.; Jing, Z.-C.; Keogh, A.M.; Langleben, D.; Kilama, M.O.; Fritsch, A.; et al. Riociguat for the treatment of pulmonary arterial hypertension. N. Engl. J. Med. 2013, 369, 330-340. [CrossRef] [PubMed]

24. Pieske, B.; Maggioni, A.P.; Lam, C.S.P.; Pieske-Kraigher, E.; Filippatos, G.; Butler, J.; Ponikowski, P.; Shah, S.J.; Solomon, S.D.; Scalise, A.-V.; et al. Vericiguat in patients with worsening chronic heart failure and preserved ejection fraction: Results of the SOluble guanylate Cyclase stimulatoR in heArT failurE patientS with PRESERVED EF (SOCRATES-PRESERVED) study. Eur. Heart J. 2017, 38, 1119-1127. [CrossRef]

25. Duca, F.; Aschauer, S.; Zotter-Tufaro, C.; Binder, C.; Kammerlander, A.; Börries, B.; Agis, H.; Kain, R.; Hengstenberg, C.; Mascherbauer, J.; et al. EXPRESS: Riociguat for the treatment of transthyretin cardiac amyloidosis - Data from a named patient use program in Austria. Pulm. Circ. 2019. [CrossRef]

26. Maurer, M.S.; Schwartz, J.H.; Gundapaneni, B.; Elliott, P.M.; Merlini, G.; Waddington-Cruz, M.; Kristen, A.V.; Grogan, M.; Witteles, R.; Damy, T.; et al. Tafamidis Treatment for Patients with Transthyretin Amyloid Cardiomyopathy. N. Engl. J. Med. 2018, 379, 1007-1016. [CrossRef]

27. Escher, F.; Senoner, M.; Doerler, J.; Zaruba, M.M.; Messner, M.; Mussner-Seeber, C.; Ebert, M.; Ensinger, C.; Mair, A.; Kroiss, A.; et al. When and how do patients with cardiac amyloidosis die? Clin. Res. Cardiol. 2020, 109, 78-88. [CrossRef]

28. Barrett, C.D.; Dobos, K.; Liedtke, M.; Tuzovic, M.; Haddad, F.; Kobayashi, Y.; Lafayette, R.; Fowler, M.B.; Arai, S.; Schrier, S.; et al. A Changing Landscape of Mortality for Systemic Light Chain Amyloidosis. JACC Heart Fail 2019, 7, 958-966. [CrossRef]

29. González-López, E.; Gagliardi, C.; Dominguez, F.; Quarta, C.C.; De Haro-del Moral, F.J.; Milandri, A.; Salas, C.; Cinelli, M.; Cobo-Marcos, M.; Lorenzini, M.; et al. Clinical characteristics of wild-type transthyretin cardiac amyloidosis: Disproving myths. Eur. Heart J. 2017, 38, 1895-1904. [CrossRef]

(C) 2020 by the authors. Licensee MDPI, Basel, Switzerland. This article is an open access article distributed under the terms and conditions of the Creative Commons Attribution (CC BY) license (http://creativecommons.org/licenses/by/4.0/). 